\title{
Ion dynamics driven by a strongly nonlinear plasma wake
}

\author{
V K Khudiakov ${ }^{1,2}$, K V Lotov $^{1,2}$, M C Downer ${ }^{3}$ \\ ${ }^{1}$ Budker Institute of Nuclear Physics, Novosibirsk, Russia \\ ${ }^{2}$ Novosibirsk State University, Novosibirsk, Russia \\ ${ }^{3}$ University of Texas at Austin, Austin, TX, USA \\ 28 September 2021
}

\begin{abstract}
In plasma wakefield accelerators, the wave excited in the plasma eventually breaks and leaves behind slowly changing fields and currents that perturb the ion density background. We study this process numerically using the example of a FACET experiment where the wave is excited by an electron bunch in the bubble regime in a radially bounded plasma. Four physical effects underlie the dynamics of ions: (1) attraction of ions toward the axis by the fields of the driver and the wave, resulting in formation of a density peak, (2) generation of ion-acoustic solitons following the decay of the density peak, (3) positive plasma charging after wave breaking, leading to acceleration of some ions in the radial direction, and (4) plasma pinching by the current generated during the wavebreaking. Interplay of these effects result in formation of various radial density profiles, which are difficult to produce in any other way.
\end{abstract}

Keywords: plasma wakefield acceleration, strongly nonlinear wake, ion-acoustic solitons 


\section{Introduction}

Acceleration of electrons and positrons in near-lightspeed plasma waves is a rapidly developing field of research [1-5]. The highest performing plasma accelerators are based on strongly nonlinear wakefield by a relativistic electron bunch or a high-intensity laser pulse in the blowout regime $[6,7]$. In this case, the field of the driver is strong enough to completely expel electrons from its immediate wake, forming a so-called 'bubble'.

Usually, in plasma-based accelerators, the accelerated beam (called a witness) follows closely behind the driver. The time between the two beams is too short for plasma ions to respond, except in the case of very dense electron drivers [8-12] or special regimes in which a small perturbation of the ion density stabilizes the electron driver [13]. For this reason, the ion dynamics has not attracted as much attention as other wakefield features, and has been studied mainly in the context of the wakefield lifetime [14-18] or the overall energy balance in the system [19]. However, the ion motion can result in formation of exotic, slowly changing plasma density profiles, which may be useful for diagnostics [19,20], driver guiding [1], beam stabilization [21,22], wakefield enhancement [23, 24], radiation generation [25], or making field structure favorable for positron acceleration [26-29]. Ion density valleys or peaks can appear both in strongly nonlinear $[30,31]$ and almost linear [14-18,20,32,33] regimes and for all kinds of drivers. As more dense drivers become available [34], the importance of ion dynamics will increase. Despite the visual similarity of the observed density structures, the mechanisms of their formation are different in each case. In this paper, we study the ion dynamics after the wakefield excitation by a dense electron beam in a radially bounded plasma in the bubble regime. In this regime, the driver can act on the ions not only by means of the excited wave, but also directly by its own field and by the field of the current arising in the plasma.

The paper is organized as follows. In section 2, we describe the problem under study and the methods. Then we focus our attention on four effects, some of which are specific to electron drivers in radially bounded plasmas. The first effect is that ions from some paraxial region receive a negative radial momentum and form a density peak on the axis (section 3). In our case, both the electric field of the beam and the ponderomotive force of the plasma wave are responsible for the peak formation. The second effect is the appearance of ion-acoustic solitons (section 4). The on-axis density peak eventually breaks up into several smaller, diverging peaks, which propagate at the ion-sound velocity. The third effect is related to fast electrons that appear when the bubble

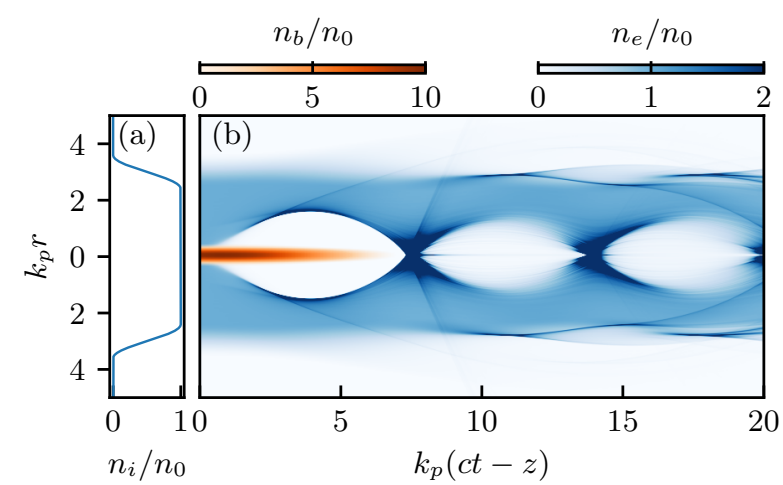

Figure 1. Wakefield excitation by the equilibrium beam: (a) radial dependence of the plasma ion density $n_{i}$ shortly after ionization and (b) the simulated density of the electron bunch $n_{b}$ (orange) and plasma electrons $n_{e}$ (blue) during the first three wakefield periods. The bunch propagates to the left.

collapses (section 5). These electrons escape from the plasma column and leave an uncompensated ion charge behind, which, in turn, pushes outer ion layers radially. The escape of fast electrons also creates a compensating current in the plasma, which ultimately leads to the fourth effect (section 6): the bulk of the ions contracts to the axis, forming a high-amplitude compression wave. In section 7 , we summarize the main findings.

\section{Statement of the problem}

We consider the case that corresponds to the recent E224 experiment [19] at the SLAC Facility for Advanced aCcelerator Experimental Tests (FACET) [35]. In the E224 experiment, the temporal evolution of the plasma density profile was measured and compared to numerical simulations. The achieved quantitative agreement proved the validity of the simulation method, so we use the same approach and the same set of parameters in our study.

An electron bunch of energy $20 \mathrm{GeV}$, charge $2 \mathrm{nC}$, root-mean-squared (rms) radius $30 \mu \mathrm{m}$ and $\mathrm{rms}$ length $55 \mu \mathrm{m}$ passes through a chamber filled with lithium vapor of atomic density $n_{0}=8 \times 10^{16} \mathrm{~cm}^{-3}$ $[36,37]$. The bunch head field-ionizes the lithium and creates a plasma column of radius about $40 \mu \mathrm{m}$ (figure 1). The plasma focuses the rest of the bunch down to the equilibrium radius of about $4 \mu \mathrm{m}$. After a short period of initial equilibration, most of the bunch propagates almost without changing its shape and excites a strongly nonlinear plasma wave in a plasma of constant radius. The equilibration stage (first $32 \mathrm{~cm}$ of bunch propagation in the plasma) is simulated with a $2 \mathrm{~d} 3 \mathrm{v}$ (axisymmetric) version of the particle-in-cell code OSIRIS [19,38]. Then, the 

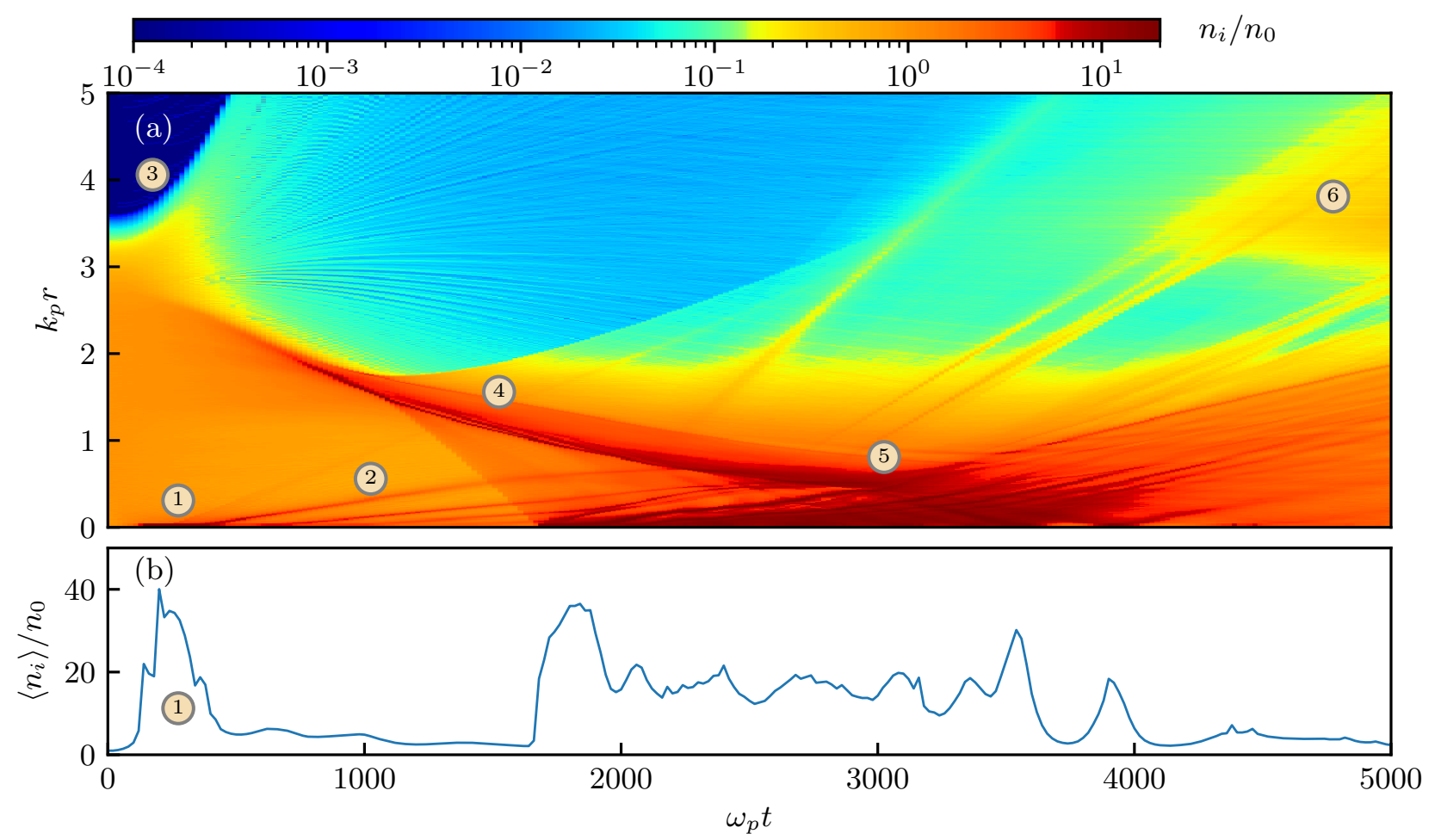

Figure 2. (a) The ion density map $n_{i}(r, t)$. The numbers in circles indicate the features discussed in the text and detailed in Supplementary Materials, movie 1. (b) The average ion density $\left\langle n_{i}\right\rangle(t)$ in the region $r<0.05 k_{p}^{-1}$. The averaged density does not depend on the simulation grid step, unlike the density on the axis.

equilibrium profiles of plasma and beam are imported into the quasistatic axisymmetric $2 \mathrm{~d} 3 \mathrm{v}$ code LCODE $[39,40]$ as initial conditions, and the simulations continue up to thousands of wakefield periods at a constant longitudinal position $z$. In the equilibrium, approximately $60 \%$ of the beam $(1.2 \mathrm{nC})$ propagates in the plasma and participates in driving the plasma wave.

To present the results in a more general form, we measure times in units of $\omega_{p}^{-1}$, distances in $k_{p}^{-1}=c / \omega_{p}$, fields in $E_{0}=m_{e} c \omega_{p} / e$, and densities in $n_{0}$, where $\omega_{p}=\sqrt{4 \pi n_{0} e^{2} / m_{e}}$ is the plasma frequency, $m_{e}$ is the electron mass, $e$ is the elementary charge, and $c$ is the speed of light. For $n_{0}=8 \times 10^{16} \mathrm{~cm}^{-3}$, $\omega_{p}^{-1} \approx 60 \mathrm{fs}, k_{p}^{-1} \approx 19 \mu \mathrm{m}$, and $E_{0} \approx 27 \mathrm{GV} / \mathrm{m} \approx$ 0.9 MGs. For lithium ions of the mass $m_{i} \approx 12850 m_{e}$, the characteristic timescale of ion response is $\tau_{i}=$ $\omega_{p}^{-1} \sqrt{m_{i} / m_{e}} \approx 113 \omega_{p}^{-1} \approx 7 \mathrm{ps}$.

The radius of the simulation window is $500 k_{p}^{-1}$, the radial grid step is $0.005 k_{p}^{-1}$, and the time step is $0.005 \omega_{p}^{-1}$. The plasma consists of two species (electrons and ions) with $2.5 \times 10^{5}$ equally weighted macro-particles in each. The number of plasma macroparticles slightly increases due to impact ionization of the surrounding neutral vapor [19], but this effect is not significant at the times considered here.

\section{On-axis density peak}

Formation of the ion density peak near the axis (figure 2, feature 1) is routinely observed in simulations of various plasma-based accelerator schemes [14-17,20, $31,32,41]$. In the bubble regime, the peak appears because of the strong radial electric field of an electron beam located inside the bubble $[8,30]$. In turn, in moderately nonlinear wakes, the inward radial force on the ions arises from specific properties of the plasma wave. The ponderomotive force of the wave is such that it pushes the plasma towards the axis $[14,15]$. Another explanation of the same effect is that plasma electrons have different oscillation amplitudes at different radii, which leads to an average charge separation and, consequently, to a nonzero radial field experienced by the ions $[16,18]$. The ions gain the radial momentum gradually over many wave periods rather than during a short time of driver passage.

In the considered case of a dense electron driver, the contributions of both the driver and the wave are important. The total charge density in the axial region is such that there is a large negative charge of the beam in the first bubble, and then narrow negatively charged and extended positively charged regions alternate (figure 3 ). The ions first receive an 


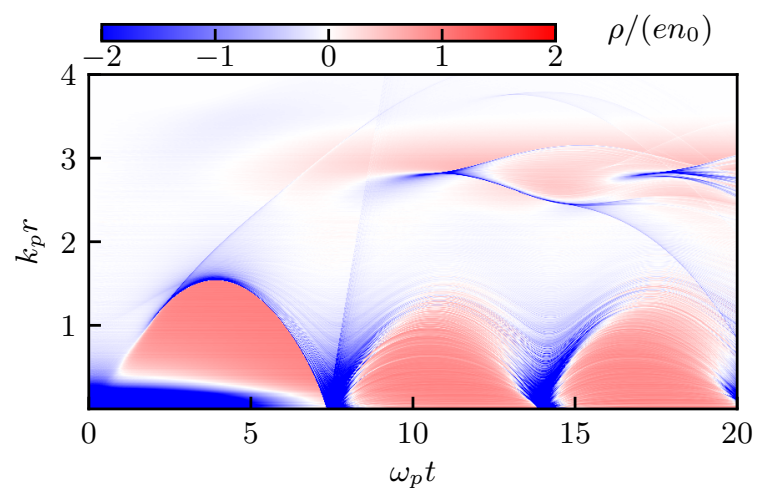

Figure 3. The total charge density of plasma and bunch, $\rho=e\left(n_{i}-n_{e}-n_{b}\right)$.

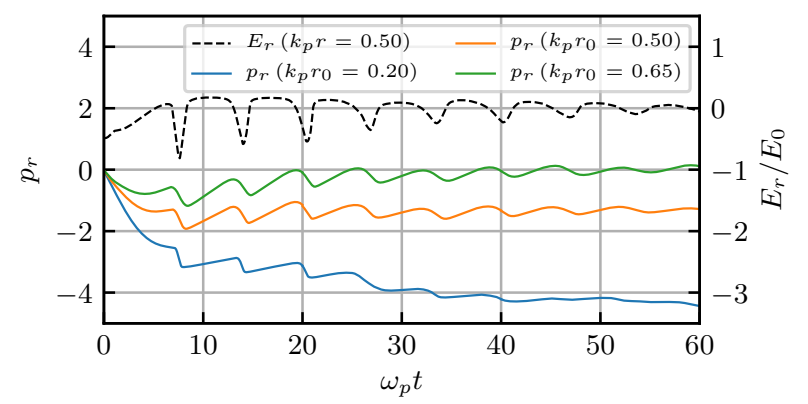

Figure 4. The radial momentum $p_{r}(t)$ of ions initially located at different radial positions $r_{0}$ (colored lines) and the radial electric field $E_{r}(t)$ at $r=0.5 k_{p}^{-1}$ (black dashed line).

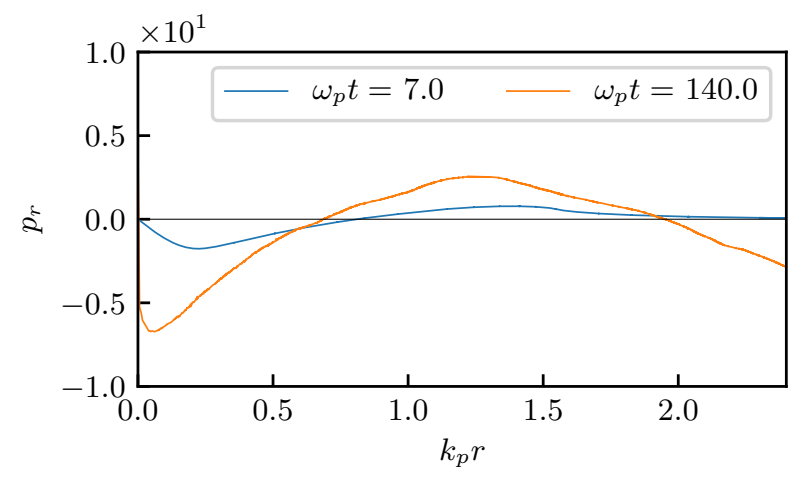

Figure 5. The radial ion momentum $p_{r}(r)$ at different times. The line $\omega_{p} t=7$ shows the momentum gained mainly because of the beam field; the line $\omega_{p} t=140$ corresponds to the arrival of near-axis ions to the axis and shows the combined effect of the beam and the wave.

inward push from the driver and then experience an oscillating force with a non-zero average (figure 4). Relative contributions of the initial push and wave force are comparable in value. In the considered case, the wave contribution is roughly 5 times stronger: the line $\omega_{p} t=7$ in figure 5 characterizes the initial push,

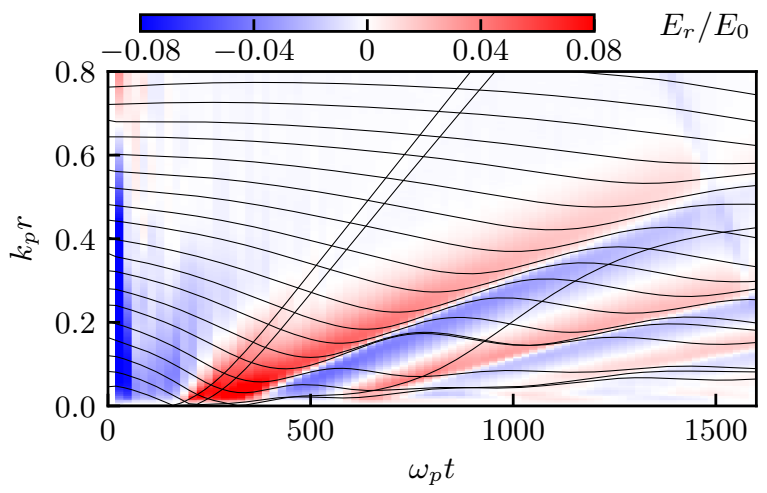

Figure 6. Equidistantly selected ion trajectories $r(t)$ plotted over the map of the radial electric field $E_{r}(r, t)$. The field is timeaveraged over $20 \omega_{p}^{-1}$ to reduce the effect of plasma oscillations.

while the difference between this line and the line $\omega_{p} t=140$ is due to the wave force. As a consequence, the time when ions from the near-axis region reach the axis is shorter than it would be if they received only the initial push, $140 \omega_{p}^{-1}$ instead of $10^{3} \omega_{p}^{-1}$. The latter number follows from the slope of the blue line in figure 5 .

\section{Ion-acoustic solitons}

The movement of the ions toward the axis, followed by the formation of a density peak there, gives rise to ionacoustic solitons, which are seen as diverging density ridges in figure 2 (feature 2). When the first ions cross the axis, they not only create the density peak, but also a positive ambipolar potential, which attracts additional electrons to maintain average plasma quasineutrality. The resulting radial electric field reverses the direction of the next portion of ions (figure 6). The counterstreaming ions form an off-axis density peak accompanied by an ambipolar potential and an electric field directed away from the peak. The peak moves radially outward against the background of inwardmoving ions (figure 7). As the soliton moves away from the axis, there is nothing to prevent the next portion of ions from passing through the soliton, reaching the axis and forming the next density peak there, which gives rise to the next soliton, and so on. Soliton generation continues as long at there is ion motion towards the axis. The dynamics of ion and electron density, ion velocity and radial electric field during soliton formation is detailed in the Supplementary materials, movie 1. Similar solitons, but not the mechanism of their formation, were also observed in simulations [31].

To make sure that the observed feature really behaves as a soliton, we compare its velocity with the velocity expected for a soliton. We follow the approach 


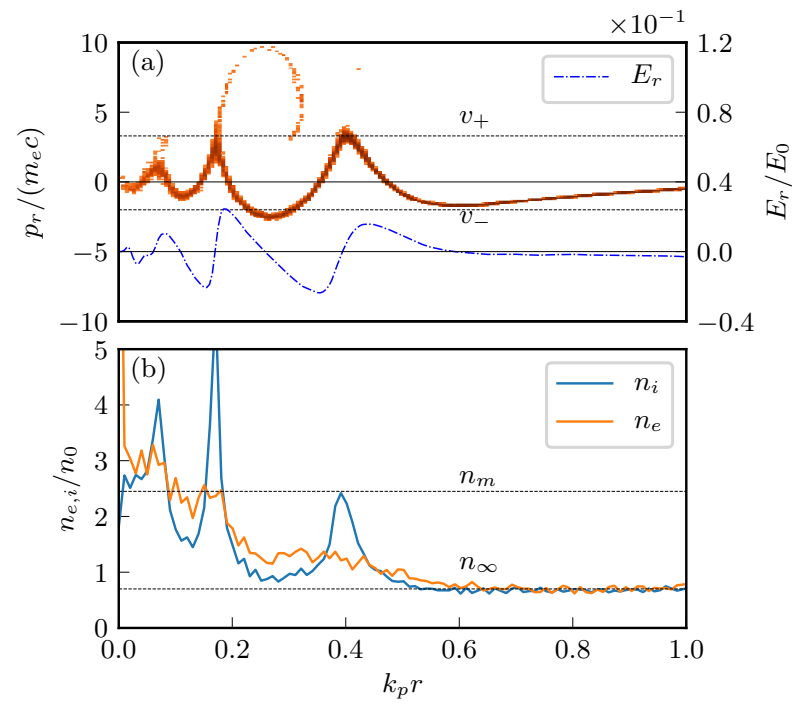

Figure 7. (a) The phase portrait of plasma ions (orange points) and the radial electric field $E_{r}$ (blue dashed line) at $\omega_{p} t=1200$. The electric field is time-averaged over $20 \omega_{p}^{-1}$ to reduce the effect of plasma oscillations. (b) The corresponding radial dependencies of electron $\left(n_{e}\right)$ and ion $\left(n_{i}\right)$ densities. The electron density is smoother because of the high electron temperature, and this leads to an ambipolar potential. The dashed lines show the soliton parameters discussed in the text.

outlined in Ref. [42] and start from the hydrodynamic equations for one-dimensional ion motion:

$$
\begin{aligned}
\frac{\partial n}{\partial t} & =-\frac{\partial(n v)}{\partial x}, \\
\frac{\partial v}{\partial t} & =-\frac{\partial}{\partial x}\left(\frac{v^{2}}{2}+\frac{\phi}{\tilde{m}_{i}}\right), \\
\frac{\partial^{2} \phi}{\partial x^{2}} & =n_{\infty} e^{\phi / T}-n,
\end{aligned}
$$

where we use dimensionless quantities: ion density $n=n_{i} / n_{0}$, electrostatic potential $\phi$ (normalized to $\left.m_{e} c^{2} / e\right)$, ion velocity $v=v_{i} / c$, unperturbed ion density $n_{\infty}$, electron temperature $T=T_{e} /\left(m_{e} c^{2}\right)$, and ion mass $\tilde{m}_{i}=m_{i} / m_{e}$. The ions are cold.

We are looking for a stationary solution, vanishing at infinity, propagating with velocity $u$ and depending on $\zeta=x-u t$. Then the equations can be integrated:

$$
\begin{aligned}
& n=\frac{n_{\infty}}{\sqrt{1-\phi / \phi_{0}}}=\frac{n_{\infty} u}{u-v}, \\
& v=u\left(1-\sqrt{1-\phi / \phi_{0}}\right)=\frac{n-n_{\infty}}{n} u, \\
& \left(\frac{\partial \phi}{\partial \zeta}\right)^{2}=2 n_{\infty}\left(T\left(e^{\phi / T}-1\right)+2 \phi_{0}\left(\sqrt{1-\phi / \phi_{0}}-1\right) d 6,\right.
\end{aligned}
$$

where $\phi_{0}=\tilde{m}_{i} u^{2} / 2$.

Let $n_{m}, v_{m}$ and $\phi_{m}$ be the maximum values of the solution reached where $\partial \phi / \partial \zeta=0$. They are related as follows:

$\phi_{m}=\phi_{0} \frac{n_{m}^{2}-n_{\infty}^{2}}{n_{m}^{2}}$

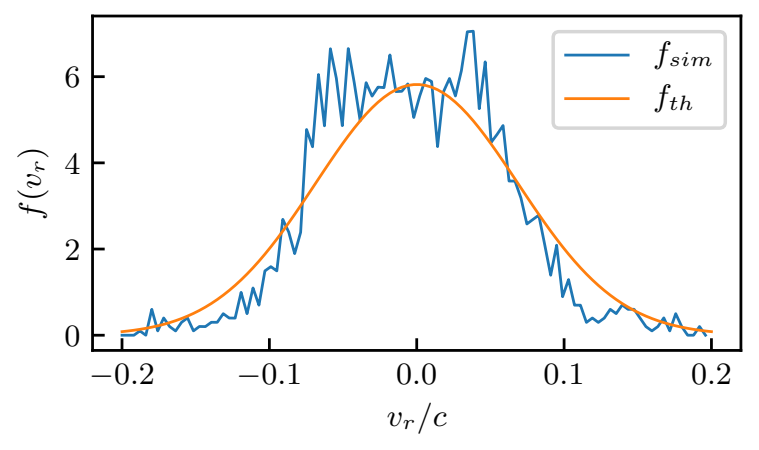

Figure 8. The electron distribution function $f_{\text {sim }}\left(v_{r}\right)$ observed in simulations near the outermost soliton at $\omega_{p} t=1200$ and the Maxwell distribution $f_{t h}$ for the estimated temperature $T_{e}=2.4 \mathrm{keV}$.

$T\left(e^{\phi_{m} / T}-1\right)+2 \phi_{0}\left(\sqrt{1-\phi_{m} / \phi_{0}}-1\right)=0$.

We analyze the outermost soliton, as its properties are least affected by the cylindricity. Its numerical parameters can be taken from figure 7: background ion velocity $v_{-}=-1.6 \times 10^{-4}$, maximum ion velocity $v_{+}=2.6 \times 10^{-4}$, background density $n_{\infty}=0.7$, and peak ion density $n_{m}=2.45$. From the equation (5), we find the soliton propagation velocity with respect to the ion background

$u=\frac{n_{m}}{n_{m}-n_{\infty}}\left(v_{+}-v_{-}\right)=6.0 \times 10^{-4}$

and in the laboratory frame

$u_{l}=u+v_{-}=4.2 \times 10^{-4}$.

On the other hand, the propagation velocity of the density peak can be directly measured in figure 2(a):

$u_{l, \text { sim }}=4.4 \times 10^{-4}$,

which agrees with the theoretical value (10).

The velocity and peak density of the soliton determine the maximum potential and electron temperature from the equation (8): $\phi_{m}=3.9 \times 10^{-3}$, $T=4.7 \times 10^{-3}, T_{e}=2.4 \mathrm{keV}$. The ion sound velocity for this temperature is close to the soliton velocity (9):

$c_{s}=\sqrt{\frac{T_{e}}{m_{i}}}=6.1 \times 10^{-4} c$.

The Maxwell distribution for this temperature is close to the electron velocity distribution observed in simulations (figure 8). Taken together, this proves that the observed features are ion-acoustic solitons.

\section{Wave breaking}

The wavebreaking in the considered strongly nonlinear regime leads to ejection of some electrons from the plasma with high (relativistic) velocities (figure 9). These electrons acquire a large momentum when the 

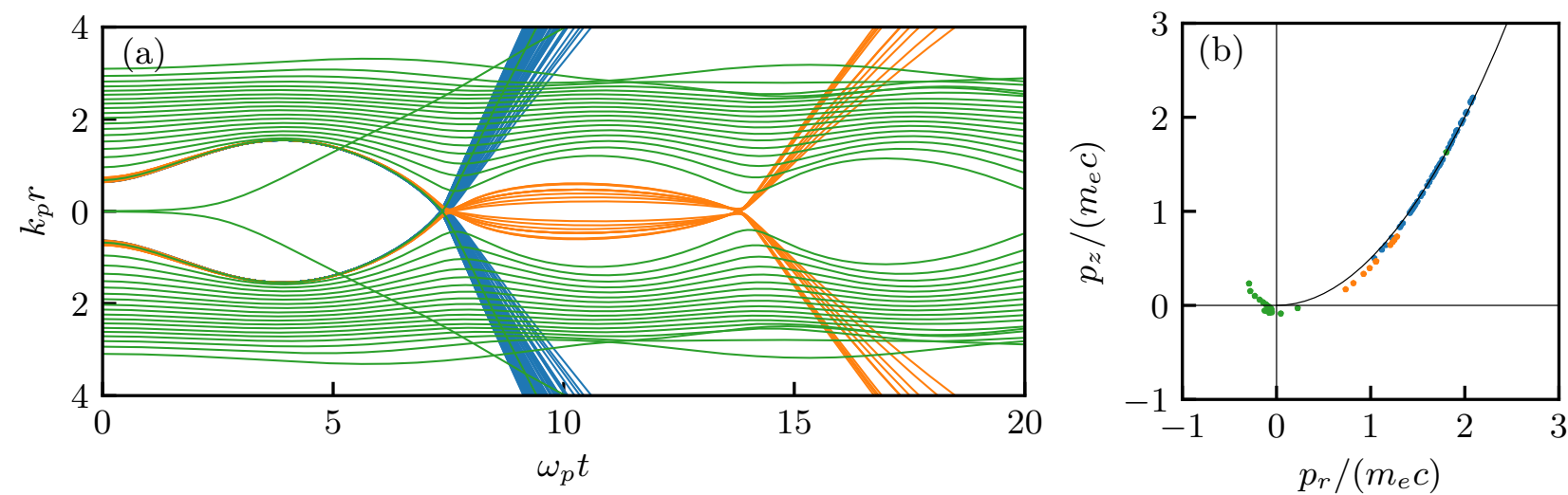

Figure 9. (a) Selected trajectories of plasma electrons in the co-moving window: blue (orange) lines correspond to electrons escaping after the first (second) bubble, and green lines to other electrons. (b) The position of these electrons at $\omega_{p} t=18$ on the momentum plane $\left(p_{r}, p_{z}\right)$. The thin line shows the parabola $p_{z}=p_{r}^{2} /\left(2 m_{e} c\right)$.

bubbles collapse and escape from the plasma column in the form of diverging tail waves. Other types of wavebreaking discussed in Ref. [43] do not result in such high energies. Most of the high-energy electrons appear in the tail of the first bubble. They carry away about $40 \%$ of the wakefield energy and form a density ridge visible on the electron density map (figure 1). Subsequent bubbles also generate tail waves, but weaker ones. The electrons ejected from different bubbles are initially located at approximately the same radii (figure $9(\mathrm{a})$ ).

After the high-energy electrons leave the plasma, the excess positive charge concentrates in its outer layer (figure 3) and generates a radial electric field around the plasma and inside this layer (figure 10(a)). The layer thickness (of the order of $k_{p}^{-1}$ ) is much greater than the Debye length corresponding to the electron temperature [44], because the near-boundary electrons oscillate (figures 1 and 3) and respond to the average field as if their temperature were high. The ions located in this layer are then accelerated in the radial direction, forming the feature 3 in figure 2. As the plasma expands radially, the region of the strong electric field also moves to larger radii (figure 10(b)). Similar processes occur when a moderately nonlinear plasma wave breaks $[45,46]$ or the plasma is heated by a strong laser pulse [47].

The escaping high-energy electrons also have a large positive longitudinal momentum, that is, they move in the direction of driver propagation. This is a common feature of wavebreaking, which follows from the basic wakefield properties. If the driver evolves slowly and propagates in an unperturbed plasma where the particles are initially in rest, then all plasma properties depend on the longitudinal coordinate $z$ and time $t$ only in their combination $\xi=z-c t$, and there is a relation between the relativistic factor $\gamma$ and the longitudinal momentum $p_{z}$ of plasma electrons [48]

$(\gamma-1) m_{e} c^{2}-e \Phi-c p_{z}=0$

where $\Phi$ is the so-called wakefield potential or pseudopotential related to the longitudinal electric field $E_{z}$ as

$\Phi(r, \xi)=\int_{\xi}^{\infty} E_{z}\left(r, \xi^{\prime}\right) d \xi^{\prime}$.

By introducing

$W=\frac{e \Phi}{m_{e} c^{2}}+1$

we obtain from equation (13)

$p_{z}=\frac{p_{r}^{2}}{2 m_{e} c W}+\frac{1-W^{2}}{2 W}$.

Outside the region of strong wakefields, $e \Phi \ll m_{e} c^{2}$, and $W \approx 1$. Therefore, any electron escaping from the plasma with a large radial momentum $p_{r}$ must also have a large positive longitudinal momentum

$p_{z} \approx \frac{p_{r}^{2}}{2 m_{e} c}$,

and simulations confirm this (figure 9(b) and movie 2 in Supplementary materials).

\section{Plasma pinching}

As forward-moving fast electrons leave the plasma column, an average current of the opposite direction appears inside the plasma. This current creates a strong azimuthal magnetic field (figure 10(c),(d)), which in turn exerts an inward radial force on the return current of plasma electrons. A charge separation electric field (blue area at $\omega_{p} t \lesssim 500$ in figure 10(b)) transmits this force to ions and accelerates them toward the axis. Thus, most of the ions move inward 



Figure 10. Evolution of (a),(b) radial electric field $E_{r}$ and (c),(d) azimuthal magnetic field $B_{\phi}$ at different timescales. At the longer timescale, the fields are time-averaged over $20 \omega_{p}^{-1}$.

under the magnetic force, while a smaller (outer) part moves outward, entrained by the escaping fast electrons (figure 2(a)). The inward-directed force is nonlinear in radius and is strongest at the periphery, so the far ions overtake the near ones and form a compression wave (feature 4 in figure 2(a)), which behaves like a soliton and is similarly accompanied by charge-separation electric fields (figure 10(b)). When high density spikes appear in the near-axis region, they generate ion-acoustic solitons by the mechanism discussed in section 4. As a result, the dense plasma pinch formed near the axis is highly inhomogeneous (feature 5 in figure $2(\mathrm{a})$ ).

At even longer times, ionization of the surrounding neutrals occurs, and we observe an increase of ion density beyond the initial plasma radius (feature 6 in figure 2(a)). We will not discuss this feature, as it is described in detail in Ref. [19].

\section{Discussion}

The technique in which an additional beam or discharge passes through the plasma before the main pulse to form the desired density profile is widely used in plasma-based wakefield accelerators [1]. The longterm evolution of a strongly nonlinear plasma wave can provide additional opportunities for this. We

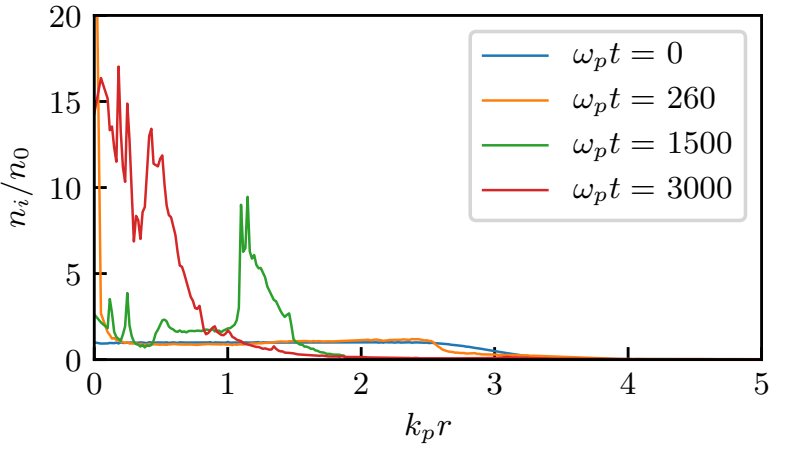

Figure 11. Radial profiles of the ion density at different times after the drive beam passage.

considered the case typical of a high-amplitude plasma wave in a finite-radius plasma and observed a variety of ion density profiles formed at different times after beam passage (figure 11). These are a narrow density spike at the center of a nearly uniform plasma at $\omega_{p} t \sim 260$, a density channel with steep high-density walls at $\omega_{p} t \sim 1500$, and a narrow filament with an order of magnitude increased density at $\omega_{p} t \sim 3000$. A finescale density structure is superimposed on the latter two. At the heart of this variety are the four effects discussed. Two of them, the on-axis density peaking 
and the formation of ion-acoustic solitons, do not require a boundary and will also work in an unbounded plasma. Two others, the radial acceleration of sheath ions and the plasma pinching, are characteristic only of radially bounded plasmas.

\section{Acknowledgments}

The authors thank T. Silva and J. Vieira for providing the OSIRIS output data from [19], which were used as input data for the simulations presented, and thank M. Hogan, A. Sosedkin, V. Yakimenko, and R. Zgadzaj for helpful discussions. This work was supported by the Russian Science Foundation, project 20-12-00062.

\section{References}

[1] E. Esarey, C. B. Schroeder, and W. P. Leemans, Rev. Mod. Phys. 81, 1229 (2009).

[2] M.J.Hogan, Reviews of Accelerator Science and Technology 9, 63 (2016).

[3] E. Adli and P. Muggli, Reviews of Accelerator Science and Technology 9, 85 (2016).

[4] M.C. Downer, R. Zgadzaj, A. Debus, U. Schramm, and M.C. Kaluza, Rev. Mod. Phys. 90, 035002 (2018).

[5] F. Albert, et al., New J. Phys. 23, 031101 (2021).

[6] J.B.Rosenzweig, B.Breizman, T.Katsouleas, and J.J.Su Phys. Rev. A 44, R6189 (1991).

[7] A.Pukhov, J. Meyer-ter-Vehn, Appl. Phys. B 74, 355 (2002).

[8] J.B.Rosenzweig, A. M.Cook, A.Scott, M.C.Thompson, and R.B.Yoder, Phys. Rev. Lett. 95, 195002 (2005).

[9] R. Gholizadeh, T. Katsouleas, P. Muggli, C. Huang, and W. Mori, Phys. Rev. Lett. 104, 155001 (2010).

[10] R. Gholizadeh, T. Katsouleas, C. Huang, W.B. Mori, and P. Muggli, Phys. Rev. ST Accel. Beams 14, 021303 (2011)

[11] W. An, W.Lu, C. Huang, X. Xu, M.J. Hogan, C. Joshi, and W.B. Mori, Phys. Rev. Lett. 118, 244801 (2017).

[12] C. Benedetti, C.B. Schroeder, E. Esarey, and W.P. Leemans, Phys. Rev. Accel. Beams 20, 111301 (2017).

[13] T.J. Mehrling, C. Benedetti, C.B. Schroeder, E. Esarey, and W.P. Leemans, Phys. Rev. Lett. 121, 264802 (2018).

[14] L.M.Gorbunov, P.Mora, and A.A.Solodov, Phys. Rev. Lett. 86, 3332 (2001).

[15] L.M.Gorbunov, P.Mora, and A.A.Solodov, Phys. Plasmas 10, 1124 (2003).

[16] J.Vieira, R.A.Fonseca, W.B.Mori, and L.O.Silva, Phys. Rev. Lett. 109, 145005 (2012)

[17] R.I. Spitsyn, I.V. Timofeev, A.P. Sosedkin, and K.V. Lotov, Phys. Plasmas 25, 103103 (2018).

[18] J. Vieira, R. A. Fonseca, W. B. Mori, and L. O. Silva, Phys. Plasmas 21, 056705 (2014).

[19] R.Zgadzaj, et al., Nat. Comm. 11, 4753 (2020).

[20] M.F. Gilljohann, et al., Phys. Rev. X 9, 011046 (2019).

[21] C.B. Schroeder, E. Esarey, C. Benedetti, and W.P. Leemans, Phys. Plasmas 20, 080701 (2013).

[22] A. Pukhov and J.P. Farmer, Phys. Rev. Lett. 121, 264801 (2018).

[23] A. Pukhov, O. Jansen, T. Tueckmantel, J. Thomas, and I.Yu. Kostyukov, Phys. Rev. Lett. 113, 245003 (2014).

[24] V.A.Minakov, A.P.Sosedkin, and K.V.Lotov, Plasma Phys. Control. Fusion 61, 114003 (2019).

[25] G. Stupakov, Phys. Plasmas 24, 113110 (2017).

[26] K.V.Lotov, Phys. Plasmas 14, 023101 (2007).

[27] L.Yi, et al., Scientific Reports 4, 4171 (2014).
[28] Y.Li, G.Xia, K.V.Lotov, A.P.Sosedkin, and Y.Zhao, Plasma Phys. Control. Fusion 61, 025012 (2019).

[29] S. Diederichs, T.J. Mehrling, C. Benedetti, C.B. Schroeder, A. Knetsch, E. Esarey, and J. Osterhoff, Phys. Rev. Accel. Beams 22, 081301 (2019).

[30] K.I. Popov, W. Rozmus, V.Yu. Bychenkov, N. Naseri, C.E. Capjack, and A.V. Brantov, Phys. Rev. Lett. 105, 195002 (2010).

[31] A.A.Sahai, Phys. Rev. Accel. Beams 20, 081004 (2017).

[32] V.A. Balakirev, V.I. Karas', and I.V. Karas', Plasma Physics Reports 28, 125 (2002).

[33] S. Kar, et al., New Journal of Physics 9, 402 (2007).

[34] V. Yakimenko, L. Alsberg, E. Bong, G. Bouchard, C. Clarke, C. Emma , S. Green , C. Hast, M.J. Hogan, J. Seabury, et al., Phys. Rev. Accel. Beams 22, 101301 (2019).

[35] M.J.Hogan, et al., New Journal of Physics 12, 055030 (2010).

[36] P. Muggli, K.A. Marsh, S. Wang, C.E. Clayton, S. Lee, T.C. Katsouleas, and C. Joshi, IEEE Trans. Plasma Sci. 27, 791 (1999).

[37] M.Litos, et al., Nature 515, 92 (2014).

[38] R.A. Fonseca, J. Vieira, F. Fiuza, A. Davidson, F.S. Tsung, W.B. Mori, and L.O. Silva, Plasma Phys. Control. Fusion 55, 124011 (2014).

[39] K.V.Lotov, Phys. Rev. ST Accel. Beams 6, 061301 (2003).

[40] A.P.Sosedkin, K.V.Lotov, Nuclear Instr. Methods A 829, $350(2016)$

[41] A. Lifschitz, F. Sylla, S. Kahaly, A. Flacco, M. Veltcheva, G. Sanchez-Arriaga, E. Lefebvre, and V. Malka, New J. Phys. 16, 033031 (2014).

[42] R.K. Dodd, J.C. Eilbeck, J.D. Gibbon, and H.C. Morris, Solitons and Nonlinear Wave Equations, Academic Press, London, 1982.

[43] J. Luo, M. Chen, G.-B. Zhang, T. Yuan, J.-Y. Yu, Z.-C. Shen, L.-L. Yu, S.-M. Weng, C. B. Schroeder, and E. Esarey, Phys. Plasmas 23, 103112 (2016).

[44] A.V. Gurevich, L.V. Pariiskaya, and L.P. Pitaevskii, Sov. Phys. JETP 22, 449 (1966).

[45] K.V.Lotov, A.P.Sosedkin, A.V.Petrenko, Phys. Rev. Lett. 112, 194801 (2014).

[46] R.I. Spitsyn and K.V. Lotov, Plasma Phys. Control. Fusion 63, 055002 (2021).

[47] A.Macchi, M.Borghesi, M.Passoni, Rev. Mod. Phys. 85, 751 (2013).

[48] P.Mora and T.M.Antonsen, Phys. Plasmas 4, 217 (1997). 